Probability, Networks and Algorithms wireless ad-hoc network

F. Roijers, J.L. van den Berg, M.R.H. Mandjes 
Centrum voor Wiskunde en Informatica (CWI) is the national research institute for Mathematics and Computer Science. It is sponsored by the Netherlands Organisation for Scientific Research (NWO).

CWI is a founding member of ERCIM, the European Research Consortium for Informatics and Mathematics.

CWI's research has a theme-oriented structure and is grouped into four clusters. Listed below are the names of the clusters and in parentheses their acronyms.

\section{Probability, Networks and Algorithms (PNA)}

Software Engineering (SEN)

Modelling, Analysis and Simulation (MAS)

Information Systems (INS)

Copyright (C) 2007, Stichting Centrum voor Wiskunde en Informatica

P.O. Box 94079, 1090 GB Amsterdam (NL)

Kruislaan 413, 1098 SJ Amsterdam (NL)

Telephone +31205929333

Telefax +31205924199

ISSN 1386-3711 


\title{
Fluid-flow modeling of a relay node in an IEEE 802.11 wireless ad-hoc network
}

\begin{abstract}
Wireless ad-hoc networks are based on shared medium technology where the nodes arrange access to the medium in a distributed way independent of their current traffic demand. This has the inherent drawback that a node that serves as a relay node for transmissions of multiple neighboring nodes is prone to become a performance "bottleneck". In the present paper such a bottleneck node is modeled via an idealized fluid-flow queueing model in which the complex packet-level behavior (mac) is represented by a small set of parameters. We extensively validate the model by ad-hoc network simulations that include all the details of the widely used ieee 802.11 mac-protocol. Further we show that the overall flow transfer time of a multi-hop flow, which consists of the sum of the delays at the individual nodes, improves by granting a larger share of the medium capacity to the bottleneck node. Such alternative resource sharing strategies can be enforced in real systems by deploying the recently standardized ieee $802.11 \mathrm{e}$ mac-protocol.We propose a mapping between the parameter settings of ieee $802.11 \mathrm{e}$ and the fluid-flow model, and validate the fluid-flow model and the parameter mapping with detailed system simulations.
\end{abstract}

2000 Mathematics Subject Classification: 68M10, 60K25

Keywords and Phrases: Wireless, ad hoc networks, fluid flow, queueing

Note: Work done while Frank Roijers was still with CWI. This work has been carried out partly in the SenterNovem funded project Easy Wireless and the Dutch Bsik-BRICKS project. 



\title{
Fluid-flow modeling of a relay node in an IEEE 802.11 wireless ad-hoc network
}

\author{
Frank Roijers ${ }^{1,2,3}$, Hans van den Berg ${ }^{1,4}$, and Michel Mandjes ${ }^{2,3}$ \\ 1 TNO Information and Communication Technology, the Netherlands \\ 2 Korteweg-de Vries Institute, University of Amsterdam, the Netherlands \\ 3 Centre for Mathematics and Computer Science, the Netherlands \\ 4 Department of Design and Analysis of Communication Systems, \\ University of Twente, the Netherlands
}

\begin{abstract}
Wireless ad-hoc networks are based on shared medium technology where the nodes arrange access to the medium in a distributed way independent of their current traffic demand. This has the inherent drawback that a node that serves as a relay node for transmissions of multiple neighboring nodes is prone to become a performance "bottleneck". In the present paper such a bottleneck node is modeled via an idealized fluid-flow queueing model in which the complex packet-level behavior (MAC) is represented by a small set of parameters. We extensively validate the model by ad-hoc network simulations that include all the details of the widely used IEEE 802.11 MAC-protocol. Further we show that the overall flow transfer time of a multi-hop flow, which consists of the sum of the delays at the individual nodes, improves by granting a larger share of the medium capacity to the bottleneck node. Such alternative resource sharing strategies can be enforced in real systems by deploying the recently standardized IEEE $802.11 \mathrm{E}$ MAC-protocol. We propose a mapping between the parameter settings of IEEE $802.11 \mathrm{E}$ and the fluid-flow model, and validate the fluid-flow model and the parameter mapping with detailed system simulations.
\end{abstract}

\section{Introduction}

Developments in wireless communication technology open up the possibility of deploying wireless ad-hoc networks; these networks can be rolled out instantly without any fixed infrastructure or pre-advanced configuration. Currently, IEEE 802.11 wireless LAN $[8]$ is the most popular technology used for wireless ad-hoc networks. Up to now mainly IEEE 802.11B was used for ad-hoc networking, but in 2005 IEEE $802.11 \mathrm{E}$ was standardized allowing for service differentiation.

Ad-hoc networks have two important characteristics: i) stations that cannot directly communicate with each other use other stations as relay nodes; ii) stations contend for access to the wireless medium in a distributed fashion. A consequence of the first characteristic is that certain nodes, in particular nodes that have a central location, are likely to become relay nodes having considerably higher traffic loads than other nodes. The second characteristic entails 
that there is a lack of coordination between the nodes which may result in non-optimal sharing of the medium capacity. Therefore, a relay node can easily become a performance bottleneck. For example, when a relay node obtains the same share of the medium capacity as each of its active neighboring nodes, the input rate of traffic into the relay node regularly exceeds the output rate when more than one neighboring node sends traffic via the relay node. This results in the accumulation of backlogged traffic and consequently in increasing delays.

The vast majority of ad-hoc network performance studies available in the literature is based on simulation, see e.g. [6,7]. These studies usually capture great detail of the ad-hoc network protocols, but have the intrinsic drawback that they do not provide deeper understanding of the impact of the parameters on the realized performance. Moreover, simulation runtime may become prohibitively large, hampering, e.g., sensitivity analysis or parameter optimization. Analytical performance models usually capture less detail in order to retain tractability, but do provide insight into the behavior of the system.

In [1] we introduced a performance model for 2-hop flows (i.e., file transfer) relayed by a bottleneck node in an IEEE $802.11 \mathrm{~B}$ ad-hoc network. The modeling approach is based on the principle of separation of time scales. The entire packetlevel behavior is captured in the net medium capacity, which can be obtained by the well-known model of Bianchi [2]. Subsequently the net medium capacity is used as service capacity in a flow-level model that captures the "user dynamics", i.e., the initiation and completion of file transfer from neighboring source nodes to destinations via the bottleneck node. Another aspect of the modeling approach in [1] is that the flow-level model is based on so-called fluid flows, i.e., flows are modeled as if they continuously send traffic instead of sending individual packets. This modeling approach has been successfully used before for the single-hop case, see $[5,10]$; the model in [1] could be regarded as an extension to the situation of an additional hop that requires a share of the medium capacity.

The time-scale separation considerably reduces the complexity of the model in [1]. In particular, insightful, explicit formulas are obtained for the mean values of, e.g., the delay per hop and the overall transfer time of a 2-hop flow. These expressions can easily be evaluated in order to generate numerical results. However, [1] did not investigate whether the model really accurately describes the behavior of an IEEE 802.11 ad-hoc network. Furthermore, [1] focuses on the situation where the relay node obtains the same share of the medium capacity as its neighboring nodes. An extension to improve the overall flow transfer time is the option of granting a larger share of the medium capacity to the relay node than to each of the neighboring nodes.

Contribution The present paper elaborates on the fluid-flow model, for short the fluid model, proposed in [1]. The present paper fills both above-mentioned gaps.

The first contribution is a validation of the fluid model for "equal resource sharing" of the medium capacity, i.e., the case where the bottleneck node can 
only obtain the same share of the medium capacity as each of its neighboring nodes. This model is validated for network nodes that operate according to the IEEE 802.11B MAC-protocol. The analytical results of [1], as summarized in Section 3.2, are numerically evaluated and compared to results obtained by a wireless ad-hoc network simulator with a detailed implementation of the IEEE 802.11 protocols.

The second contribution is the extension of the fluid model to an ad-hoc network scenario with so-called "unequal resource sharing" between the bottleneck and source nodes, i.e., the bottleneck node may obtain a larger share of the medium capacity. Importantly, we demonstrate that unequal resource sharing can considerably improve the transfer time of a flow. The fluid model with unequal resource sharing is validated by the earlier-mentioned IEEE $802.11 \mathrm{E}$ MAC-protocol, which can be used to implement the unequal resource-sharing policy.

Organization This paper is organized as follows. In Section 2 we introduce the ad-hoc network scenario and the fluid model of a bottleneck node considered in this paper. Section 3 validates the fluid model for an equal resource sharing IEEE $802.11 \mathrm{~B}$ bottleneck node; we obtain the service capacity used in the fluid model, present an excerpt of the analytical results and validate the fluid model by comparing it with ad-hoc network simulations. In Section 4 it is shown that unequal resource sharing between source and bottleneck nodes improves the overall flow transfer time. Section 5 presents the implementation of unequal resource sharing by an IEEE $802.11 \mathrm{E}$ bottleneck node; first we obtain the input parameters of the fluid model and second we validate this model. Finally, Section 6 concludes this paper and discusses some directions for further research.

\section{Ad-hoc network scenario and fluid-model description}

Section 2.1 introduces the ad-hoc network scenario considered in this paper. In Section 2.2 we present our fluid model that corresponds to this scenario. Section 2.3 briefly introduces the concept of the IEEE $802.11 \mathrm{E}$ EDCA-protocol.

\subsection{The ad-hoc network scenario}

As mentioned in the introduction, one of the goals of the present paper is to investigate whether the fluid-flow model, which we introduced in [1], accurately describes the behavior of a bottleneck node, both under equal and unequal resource-sharing policies. For that purpose we focus on a simple, special case of a wireless ad-hoc network: a two-hop ad-hoc network consisting of a number of source nodes that initiate flow transfers at random time instants, and a single relay node that forwards the traffic generated by the sources to the next-hop destination nodes, cf. Figure 1. The source nodes and destination nodes are all within the transmission range of the bottleneck node; the source and destination nodes are within each others sensing range, hence there are no hidden nodes. 


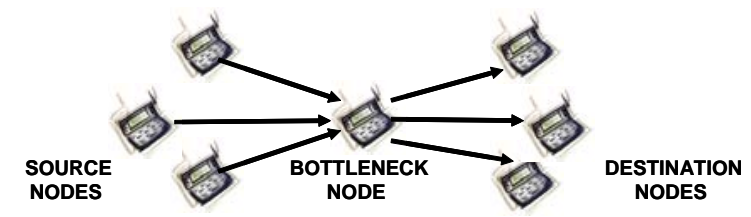

Fig. 1. Two-hop network with a single (bottleneck) node used as relay node by many sources.

The equal and unequal resource sharing policies correspond to data transmissions that are controlled by respectively the IEEE $802.11 \mathrm{~B}$ DCF ([8]) and the IEEE 802.11E EDCA ([9], also see Section 2.3) mechanisms.

\subsection{Fluid-model description}

The ad-hoc network scenario described above is now modeled as a fluid-flow queueing system. We assume a large number of source nodes which become active and initiate flow transfers to destinations via the bottleneck node according to a Poisson process with rate $\lambda$ ('flow arrival rate'). The bottleneck node relays all traffic of the source nodes in a first-come-first-served discipline. Active source nodes and the bottleneck node share the system capacity, which is conditional on the number of active source nodes $n$ and is denoted by $\mathrm{C}_{n}$. Once a source node has completed a flow transmission, the source node becomes inactive (although the last part of the flow may still be at the buffer of the bottleneck node waiting for service). Flow sizes (in terms of the amount of traffic/fluid) are i.i.d. random variables (denoted by $F$ ) with finite mean $f$ and second moment $f_{2}$. A source node has at most one flow transfer in progress.

Resource sharing between bottleneck and source nodes First we consider the case of equal resource sharing. If $n$ source nodes have a flow transfer in progress, any source node transmits its traffic (fluid) into the buffer of the bottleneck node at rate $\mathrm{C}_{n} /(n+1)$, while a rate $\mathrm{C}_{n} /(n+1)$ is used by the bottleneck node to 'serve' the buffer (i.e., to forward the traffic stored in its buffer to the next node). The amount of work backlogged in the buffer is denoted by $W_{\text {buffer }}$. In case $W_{\text {buffer }}>0$ and $n=0$ the bottleneck receives the entire capacity $\mathrm{C}_{0}$.

In case of unequal resource sharing, the maximum ratio between the share of the bottleneck node and a source node is denoted by $m_{n} \in \mathbb{R}$, and the bottleneck node may obtain capacity $m_{n} \mathrm{C}_{n} /\left(n+m_{n}\right)$. The bottleneck node will only obtain the maximum share if it can actually use it, viz. the input rate exceeds the output rate $\left(n \geq m_{n}\right)$ or if $W_{\text {buffer }}>0$. Otherwise the input and output rates are coupled, resulting in capacity share of $\mathrm{C}_{n} / 2$ for the bottleneck node. The capacity share obtained by the bottleneck node is summarized as follows:

$$
\mathrm{C}_{n} \times\left\{\begin{aligned}
m_{n} /\left(n+m_{n}\right), & \left\{W_{\text {buffer }}>0\right\} \vee\left\{n \geq m_{n}\right\}, \\
1 / 2, & \left\{W_{\text {buffer }}=0\right\} \wedge\left\{0<n<m_{n}\right\}, \\
1, & \{n=0\}
\end{aligned}\right.
$$


The source nodes always equally share the remaining capacity. Notice that IEEE 802.11 B is a special case of IEEE $802.11 \mathrm{E}$ where $m_{n}=1$.

Performance criteria Our main performance measures of interest are the steady-state buffer workload $W_{\text {buffer }}$ at the bottleneck node and the overall flow transfer time $D_{\text {overall }}$, i.e., the time required to completely transfer a flow from source to destination. The overall flow transfer time is the sum of two other performance measures: (i) the time $\left(D_{\text {source }}\right)$ a source requires to completely transfer a particular flow to the bottleneck node, and (ii) the delay of the last particle of fluid of the flow at the bottleneck node $\left(D_{\text {buffer }}^{*}\right)$.

\subsection{IEEE 802.11E Enhanced Distributed Channel Access}

IEEE $802.11 \mathrm{E}$ specifies the Enhanced Distributed channel Access (EDCA) as the distributed contention mechanism that can provide service differentiation. Whereas an 802.11B station has only one queue for all traffic, an $802.11 \mathrm{E}$ station (QSTA) has multiple queues, so-called Access Categories (ACs), and traffic is mapped into one of the ACs according to its service requirements.

Each AC contends for a Transmission OPportunity (TXOP) using the CSMA/CA mechanism (see e.g. $[8,10]$ ) using its own set of EDCA parameters values. These EDCA parameters are $\mathrm{CW}_{\min }, \mathrm{CW}_{\max }$, AIFS and the $\mathrm{TXOP}_{\text {limit }}$. The parameters $\mathrm{CW}_{\min }$ and $\mathrm{CW}_{\max }$ have the same functionality as under the DCF. The parameter AIFS (Arbitration InterFrame space) differentiates the time that each AC has to wait before it is allowed to decrement its backoff counter after the medium has become idle. Under the DCF each station has to wait for a DIFS period while the duration of an AIFS is a SIFS period extended by a discrete number of time slots AIFSN, so AIFS=SIFS+AIFSN $\times$ timeslot (where AIFSN $\geq 2$ for QSTAS). The TXOP $_{\text {limit }}$ (TXOP-limit) is the maximum duration of time that an AC may send after it has won the contention, so it may send multiple packets as long as the last

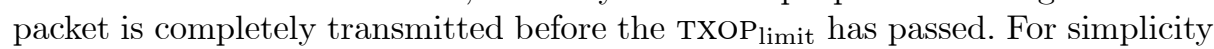
we adapt the notation that TXOP $_{\text {limit }}$ is denoted as the maximum number of packets that may be transmitted per TXOP instead of the maximum duration.

Obviously, multiple ACs can obtain the TXOP at the same moment (i.e., the backoff counters of multiple ACs reach zero at the same moment), which is called a virtual collision. Each QSTA has an internal scheduler that handles a virtual collision. The AC with the highest priority is given the TXOP and may actually initiate a transmission. The ACs of lower priority are treated as if they experienced a collision, so they have to double their contention window $\mathrm{CW}$ and start a new contention for the medium.

\section{Validation of the fluid model for IEEE 802.11B}

This section validates the fluid model with equal resource sharing as an accurate description of the behavior of the source and bottleneck nodes in an IEEE 
802.11B ad-hoc network scenario. First, we describe how to obtain accurate parameter values for the fluid model and we present a summary of the analytical results that were presented in [1]. Next, we introduce the traffic model and the ad-hoc network simulator that are used for the validation. Finally, we validate the fluid model by comparing the analytical results with simulation results of the ad-hoc network simulator.

\subsection{Mapping of IEEE 802.11B parameters}

The fluid model of Section 2.2 for equal resource sharing only requires that the capacity $\mathrm{C}_{n}$ are determined; equal resource sharing implies that $m_{n}$ equals 1 for all $n$.

The capacity $\mathrm{C}_{n}$ can be obtained by the model of Bianchi [2]. Recall that if at least one source node is active, then also the bottleneck node is active. As we assume that an active node is continuously contending for a TXOP, the ad-hoc network scenario satisfies the framework of Bianchi's model and $\mathrm{C}_{n}$ corresponds to Bianchi's saturated throughput for $n+1$ nodes. Note that we are interested in the saturated throughput at flow level (i.e., excluding all overhead), although the overheads of all osi-layers should be taken into account in the calculations.

As an example we consider an IEEE 802.11 B bottleneck node with a gross bit rate of $11 \mathrm{mbit} / \mathrm{s}$ with RTS/CTS-access. The net bit rate is $5.0 \mathrm{Mbit} / \mathrm{s}$ for all $n$, cf. the curve 802.11B in the left graph of Figure 5.

\subsection{Analysis of the fluid model}

In [1] insightful, explicit formulas for the mean values of the performance measures are presented. In the analysis it is assumed that $\mathrm{C}_{n}$ is constant for all $n$ (cf. Section 3.1), for simplicity denoted by c, which allows us to define the load of the system by $\rho=\lambda f /$ c.

The overall flow transfer time $D_{\text {overall }}$ of a flow is the sum of its flow transfer time $D_{\text {source }}$ and the buffer delay of its last particle $D_{\text {buffer }}^{*}$. Hence

$$
D_{\text {overall }}=D_{\text {source }}+D_{\text {buffer }}^{*}
$$

Notice that $D_{\text {source }}$ and $D_{\text {buffer }}^{*}$ are not statistically independent.

The mean flow transfer time $\mathbb{E} D_{\text {source }}$ is easily obtained by considering the system as a generalized processor sharing queueing model (cf. Cohen [4]) for which the stationary distribution, here denoted by $\pi_{n}$, is known; Little's law on the mean number of active source nodes yields

$$
\mathbb{E} D_{\text {source }}=\frac{\mathbb{E} N}{\lambda}=2 \frac{f / \mathrm{C}}{1-\rho} .
$$

which is insensitive to the flow-size distribution apart from its mean.

The buffer delay $D_{\text {buffer }}^{*}$ is derived from the buffer workload $W_{\text {buffer }}^{*}$ seen by the last particle, which is the sum of the workload $W_{\text {buffer }}$ upon flow arrival and 
the buffer increase $\Delta W_{\text {buffer }}$ during $D_{\text {source. }}$ The amount of work in the buffer at the bottleneck node is the difference between the total amount of work in the system $W_{\text {total }}$ (both at the sources and the buffer) and the work remaining at the source $W_{\text {sources }}$, hence

$$
\mathbb{E} W_{\text {buffer }}=\mathbb{E} W_{\text {total }}-\mathbb{E} W_{\text {sources }}=\frac{2 \rho^{2} f_{2}}{f_{\mathrm{C}}} \frac{1}{(1-2 \rho)(1-\rho)} .
$$

The expected workload increase during a flow transfer $D_{\text {source }}$ is given by

$$
\mathbb{E} \Delta W_{\text {buffer }}=\mathbb{E} D_{\text {source }}-2 f / \mathrm{C}=\frac{2 f \rho / \mathrm{C}}{1-\rho} .
$$

Therefore,

$$
\mathbb{E} W_{\text {buffer }}^{*}=\mathbb{E} W_{\text {buffer }}+\mathbb{E} \Delta W_{\text {buffer }}=\frac{2 \rho^{2} f_{2} / f_{\mathrm{C}}}{(1-2 \rho)(1-\rho)}+\frac{2 f \rho / \mathrm{C}}{1-\rho} .
$$

Observe that the buffer delay of the last particle $D_{\text {buffer }}^{*}$ is the time required to serve the amount of work $W_{\text {buffer }}^{*}$ that is present at the buffer upon arrival of the last particle. As the capacity sharing between source nodes and bottleneck node is purely processor sharing, we approximate the buffer delay of the last particle by

$$
\mathbb{E} D_{\text {buffer }}^{*} \approx \sum_{n=0}^{\infty} \pi_{n} \mathbb{E} X_{n}\left(\mathbb{E} W_{\text {buffer }}^{*}\right),
$$

where $\mathbb{E} X_{n}(\tau)$, the so-called response time for jobs in an M/M/1-PS queue presented by Coffman, Muntz, and Trotter (see [3]), is given by

$$
\mathbb{E} X_{n}(\tau)=\tau+\frac{\rho \tau}{1-\rho}+(n(1-\rho)-\rho)(f / \mathrm{C}) \frac{1-\exp (-(1-\rho) \tau \mathrm{C} / f)}{(1-\rho)^{2}} .
$$

For further details about approximation (2) we refer to [1].

\subsection{Validation scenario}

This section displays the traffic model and the ad-hoc network simulator that are used for the numerical validation in both Sections 3.4 and 5.2.

The traffic model considered in the ad-hoc network scenario is as follows. We examine the transfer of flows (files) with a mean size of 10 packets of 1500 Bytes each. The following flow-size distributions are considered: Deterministic, Exponential and Hyper-Exponential (with balanced means (e.g., see p. 359 of [12]) and a coefficient of variation (Cov) of 4 and 16). New flows are initiated according to a Poisson process and the flow initiation rate is varied between 1 and 20 .

For the fluid model the traffic parameters specified above correspond to mean flow size $f=0.12 \mathrm{mbit}$. The net capacity of the fluid model has a constant value 

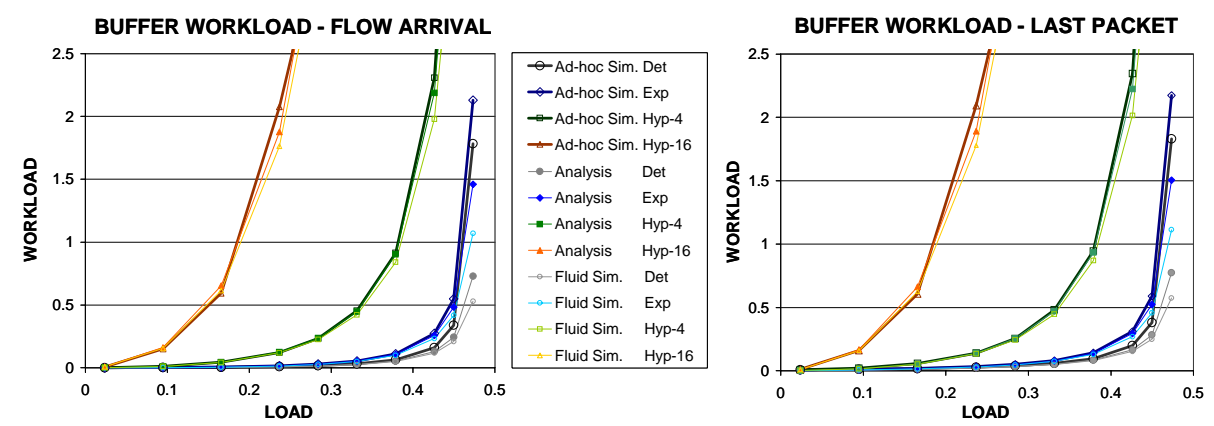

Fig. 2. Mean workload. Left: at flow arrival ( $\left.\mathbb{E} W_{\text {buffer }}\right)$. Right: last packet $\left(\mathbb{E} W_{\text {buffer }}^{*}\right)$.

of $5 \mathrm{mbit} / \mathrm{s}$; the variation of the initiation rate results in values for the load $\rho$ between 0.024 and 0.48 .

We do not consider mean flow sizes other than 10 packets, i.e., $f=0.12$, as [1] showed that the performance measures are (almost) linear in the mean flow size. Results for other mean flow sizes can be directly obtained from the results of the specified traffic model.

The ad-hoc network simulator, which is used for the numerical validation of the ad-hoc network scenario, is an own-built simulation tool in programminglanguage Delphi. All the details of CSMA/CA contention of the EDCA are included in the simulator, e.g., the back-off mechanism, physical and virtual carrier sensing, and collision handling. The PHY-layer includes propagation- and fading-models and a clear channel assessment (CCA) procedure that results in limited ranges for successfully transmitting and receiving packets and sensing transmissions of other nodes. The PHY-parameters are set according to the IEEE 802.11B standard: RTS-, CTS- and ACK-frames are transmitted at $2 \mathrm{mbit} / \mathrm{s}$ and the data-frames are transmitted at $11 \mathrm{mbit} / \mathrm{s}$.

\subsection{Numerical results}

This section numerically validates the fluid model as an accurate description of the ad-hoc network scenario of Section 2.2. The validation consists of a comparison of i) detailed simulations of ad-hoc network scenario of Section 2.1, ii) simulation of the fluid-flow model of Section 2.2, and iii) the analytical results of Section 3.2.

The graphs of Figure 2 present the mean buffer workload $\mathbb{E} W_{\text {buffer }}$ at the bottleneck node for an arbitrary packet (left) and last packet of a flow $\mathbb{E} W_{\text {buffer }}^{*}$ (right). The graphs present three curves: ad-hoc network scenario simulations, fluid-model simulations, and fluid-model analysis. In both graphs it can be seen that the three curves more or less coincide. Only for loads close to the saturation load, the results are less accurate due to the imprecision of the estimated capacity 

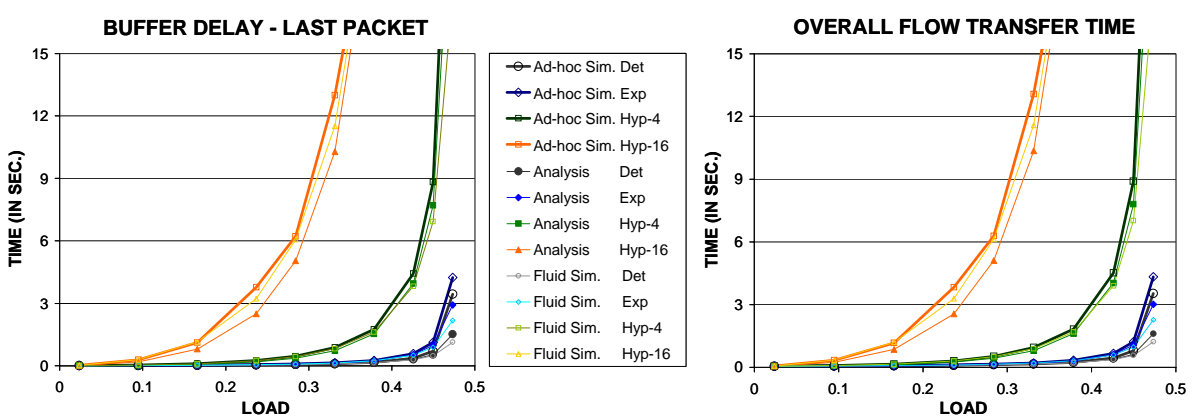

Fig. 3. Left: Mean buffer delay of the last packet/particle $\left(\mathbb{E} D_{\text {buffer }}^{*}\right)$. Right: Mean overall flow transfer time ( $\left.\mathbb{E} D_{\text {overall }}\right)$.

C. Overall the curves indicates that the fluid model accurately describes the adhoc network scenario and that the analytical results of Section 3.2 are also very good. Further, it can be observed that the buffer occupancy seen by the last particle is only slightly higher than the buffer occupancy upon flow arrival; the relatively short flow transfer time and low number of active source nodes result in a minor increase of the buffer during the flow transfer time.

Figure 3 presents the results for the mean buffer delay $\mathbb{E} D_{\text {buffer }}^{*}$ of the last packet (left) and the mean overall flow transfer time $\mathbb{E} D_{\text {overall }}$ (right). Note that the analytically obtained buffer delay of the last particle in the left graph is based on an approximation (cf. (2)). The fluid model captures the behavior of an IEEE 802.11B bottleneck very well as the model reflects both the impact of the load and flow-size distribution, except for high loads the results are less accurate. By comparing the graphs it is seen that the mean overall transfer time is almost completely determined by the buffer delay at the bottleneck node.

\section{Benefits of unequal resource sharing}

The previous section studied the model where the system capacity is equally shared amongst the active source nodes and the bottleneck node (i.e., $m_{n}=1$ ), this section studies the effects of assigning a different share of the system capacity to the bottleneck node (i.e., $m_{n} \neq 1$ ). The objective is to reduce the overall flow transfer time $D_{\text {overall }}$, which is the sum of the delays $D_{\text {source }}$ and $D_{\text {buffer }}^{*}(\mathrm{cf} .(1))$, by optimizing over $m_{n}$. Obviously the optimization is a trade-off: by granting a larger share of the capacity to the bottleneck node $D_{\text {buffer }}^{*}$ reduces while $D_{\text {source }}$ increases. We investigate the impact of the resource sharing ratio $m_{n}$.

Mathematical analysis of the fluid model with unequal resource sharing, i.e., $m_{n}>1$, is significantly harder than for the model where $m_{n}=1$. This is essentially due to the fact that in case $m_{n}>1$ the resource sharing between source nodes and bottleneck node is not completely determined by the number of active source nodes (as is the case when $m_{n}=1$ ), but also depends on the 

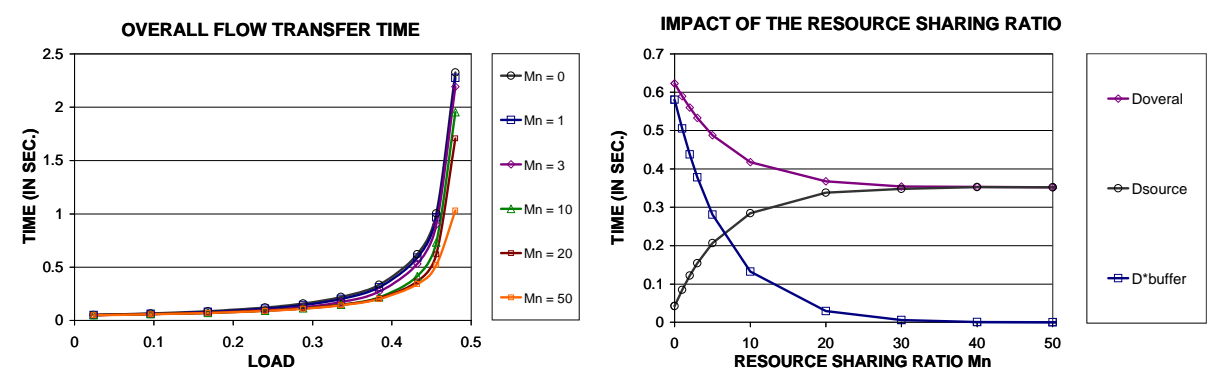

Fig. 4. Impact of resource sharing on $\mathbb{E} D_{\text {overall }}$. Left: $\mathbb{E} D_{\text {overall }}$ for different values of $m_{n}$. Right: trade-off between $\mathbb{E} D_{\text {source }}$ and $\mathbb{E} D_{\text {buffer }}^{*}$.

buffer workload $W_{\text {buffer }}$ at the bottleneck node (cf. Section 2.2). Therefore, numerical results of the fluid model for $m_{n}>1$ are obtained by simulations of the fluid model.

Figure 4 illustrates the impact of the resource sharing ratio $m_{n}$. The results are obtained by simulations of the fluid model and $m_{n}$ is independently of $n$. The left graph presents the mean overall flow transfer time $\mathbb{E} D_{\text {overall }}$ for different $m_{n}$ and loads, the right graph illustrates the trade-off between $D_{\text {source }}$ and $D_{\text {buffer }}^{*}$ for a given load (here chosen 0.43). When $m_{n}$ increases, it becomes less probable that $W_{\text {buffer }}>0$, and the bottleneck node will mostly obtain a share of $\mathrm{c} / 2$. Hence, there is hardly any queueing at the bottleneck node. From the right graph we conclude that resource sharing ratio $m_{n}=\infty$, i.e., always granting a share of $\mathrm{C} / 2$ to the bottleneck node, is optimal for the overall flow transfer time.

A special case is the resource sharing ratio $m_{n}$ is never exceeded by number of active source nodes $n$. This occurs e.g., for very large $m_{n}$ or when call admission control is applied to the maximum number of active source nodes $n_{\max }$ and $n_{\max } \leq m_{n}$. The source nodes behave as a Processor sharing model with service capacity $\mathrm{C} / 2$, flow arrival rate $\lambda$ and mean flow size $f$; hence, the mean flow transfer delay is given by (independent of the flow-size distribution) $2 f /(\mathrm{C}(1-$ $2 \rho))$.

\section{Validation of the fluid model for IEEE $802.11 \mathrm{E}$}

This section presents a validation of the fluid model for the case of unequal resource sharing. Unequal resource sharing can be achieved by an appropriate setting of the differentiating parameters in an IEEE 802.11E ad-hoc network. First, in Section 5.1 the IEEE $802.11 \mathrm{E}$ parameters are mapped onto the fluid model parameters, in particular, the capacity $\mathrm{C}_{n}$ and resource sharing ratio $m_{n}$. In Section 5.2 we validate our modeling approach by detailed ad-hoc network simulations. 

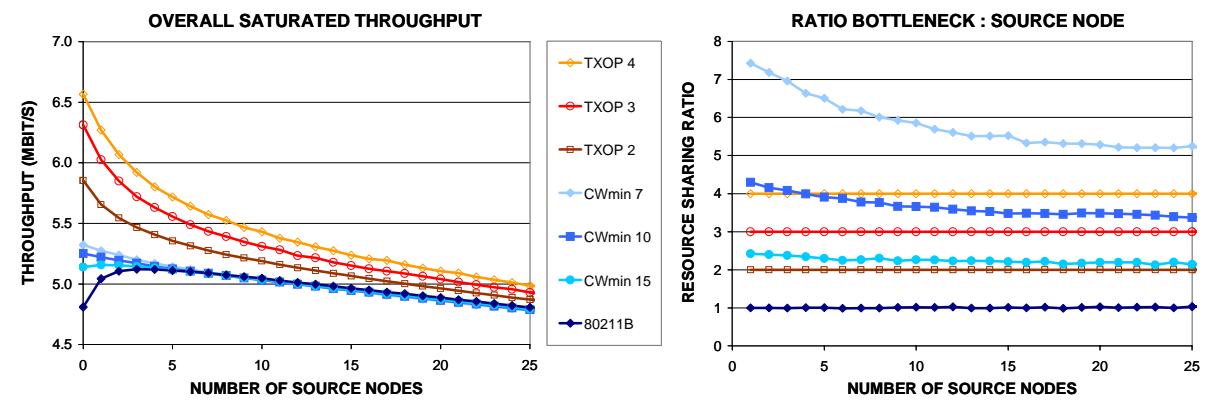

Fig. 5. Varying number of source nodes and a bottleneck node with a varying parameter setting. Left: Overall saturated throughput. Right: Resource sharing ratio.

\subsection{Mapping of IEEE 802.11E parameters}

IEEE 802.11E provides four "differentiating parameters" (cf. Section 2.3), namely $\mathrm{CW}_{\min }, \mathrm{CW}_{\max }$, AIFS, and TXOPlimit. Unfortunately, the mapping of the IEEE 802.11E parameters onto the fluid-model parameters $\mathrm{C}_{n}$ and $m_{n}$ is not selfevident, see e.g. [11].

In case the bottleneck node is saturated, i.e., $\left\{n \geq m_{n}\right\} \vee\left\{W_{\text {buffer }}>0\right\}$, the fluid model parameters $\mathrm{C}_{n}$ and $m_{n}$ can be estimated from an extension of the model of Bianchi [2] to two classes with different settings for the differentiating parameters, see e.g. [13]. In particular, the resource sharing in case of $n$ active source nodes can be obtained from the model of [13] with $n$ nodes in one class and a single node (representing our bottleneck node) in the other class. The parameters $\mathrm{C}_{n}$ and $m_{n}$ for $\left\{n \geq m_{n}\right\} \vee\left\{W_{\text {buffer }}>0\right\}$ are estimated by respectively the aggregate throughput and the ratio of the per node throughput in the two classes.

In case of a non-saturated bottleneck node, i.e., $\left\{n<m_{n}\right\} \wedge\left\{W_{\text {buffer }}=0\right\}$, the above-mentioned approach would overestimate both the non-saturated capacity $\mathrm{C}_{n}^{\prime}$ and resource sharing ratio $m_{n}^{\prime}$ due to the assumption that all nodes are saturated. Observe, the non-saturated resource sharing ratio $m_{n}^{\prime}$ equals $n$ as this resource sharing ratio couples the input rate into the bottleneck node to the output rate (cf. Section 2.2). Next, the non-saturated capacity $\mathrm{C}_{n}^{\prime}$ is estimated as follows: we consider the same differentiating parameter and its value is set such that it provides for the desired resource sharing ratio $m_{n}^{\prime}$ in the model of [13], the corresponding capacity $\mathrm{C}_{n}$ is used as an estimation of $\mathrm{C}_{n}^{\prime}$. For example, when we use differentiating parameter $\operatorname{TxOP}_{\text {limit }}=3$ and $\{n=2\} \wedge\left\{W_{\text {buffer }}=0\right\}$, then the resource sharing ratio $m_{2}^{\prime}$ equals 2 ; therefore $\mathrm{C}_{2}^{\prime}$ is estimated by $\mathrm{C}_{2}$ which is the saturated capacity for $\mathrm{TXOP}_{\text {limit }}=2$.

Figure 5 shows the saturated throughput (left graph) and the resource sharing ratio (right graph) as a function of the number of source nodes. First, we vary the value of $\mathrm{CW}_{\min }$ at the bottleneck node; all other parameters of the bottleneck node and all parameters of the source nodes are set according to the IEEE $802.11 \mathrm{~B}$ standard. Then we do a similar experiment in which we vary the 

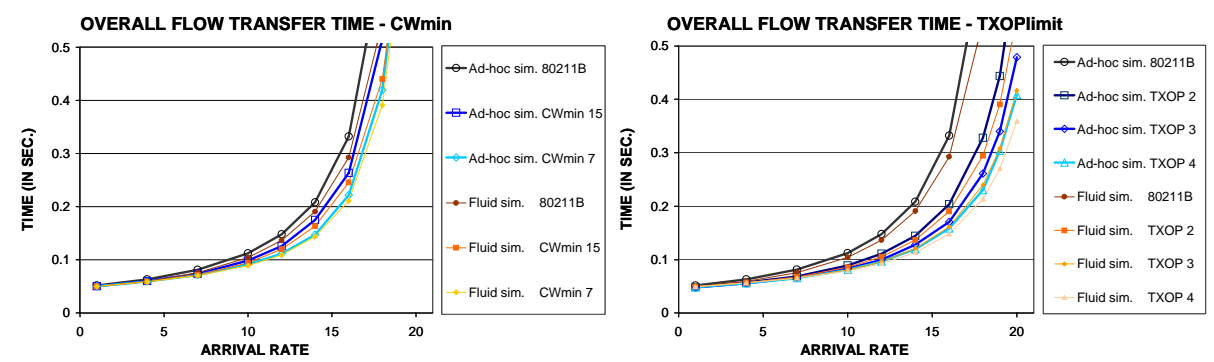

Fig. 6. System simulations and fluid-model simulations of the overall flow transfer time. Bottleneck node with varying parameter setting. Left: $\mathrm{CW}_{\min }$. Right: $\mathrm{TXOP}_{\text {limit }}$.

TXOP $_{\text {limit }}$ of the bottleneck node, while all other parameters are set according to IEEE 802.11B. The left graph illustrates that higher overall throughputs are obtained by an IEEE $802.11 \mathrm{E}$ bottleneck node, especially for parameter TXOP limit $_{\text {. }}$. In the right graph the throughput ratios for parameter $\mathrm{TXOP}_{\text {limit }}$ are trivial, the ratios for parameter $\mathrm{CW}_{\min }$ are examples of non-trivial resource sharing as, intuitively, the throughput ratio for $\mathrm{CW}_{\min }$ is the inverse of the $\mathrm{CW}_{\min }$ parametersetting ratio. For example, when $\mathrm{CW}_{\min }$ of the bottleneck node is set to 7 , then the expected ratio is $31 / 7 \approx 4$, but the realized ratio is larger than 6 for a small number of active source nodes.

\subsection{Numerical results}

In the present section the fluid model for unequal resource sharing is numerically validated by ad-hoc network simulations. The validation scenario is as in Section 3.3 and the experiments coincide with those of the previous section: one of the parameters $\mathrm{CW}_{\text {min }}$ or $\mathrm{TXOP}_{\text {limit }}$ of the bottleneck node is varied, all other parameters of the bottleneck node and all parameters of the source nodes are set according to the IEEE 802.11B standard.

For fluid-flow simulations, of which results are presented in Figure 6, we use $\mathrm{C}_{n}$ and $m_{n}$ estimated by the ad-hoc network simulator. The reason is that the fluid model is very sensitive for the used capacity if the offered load is close to the available capacity, cf. Section 3.4. Bianchi's model is proven to be accurate and the differences between results of the model and the ad-hoc network scenario simulations are small (just a few percent), but this approach ensures that deviations between the fluid model and the ad-hoc network scenario are solely due to fluid-modeling assumptions.

Figure 6 displays a comparison of ad-hoc network scenario simulations and fluid-model simulations. The fluid model simulations slightly underestimate the ad-hoc network scenario simulation results, but the behavior of the differentiating parameters is captured fairly well. The small deviations can be the result of modeling assumptions, e.g., in the fluid-model we assume that $\mathrm{C}_{n}$ and $m_{n}$ are instantly valid after the number of active source nodes has changed. By slightly 
modifying the parameter values, i.e., a minimal reduction of $\mathrm{C}_{n}$, the results coincide. We conclude that the fluid model of Section 2.2 accurately describes the behavior of unequal resource sharing in an IEEE $802.11 \mathrm{E}$ ad-hoc network.

\section{Concluding remarks and directions for further research}

In this paper we have shown that the fluid-flow model is an accurate description of multi-hop flows relayed by a performance bottleneck node in a wireless ad-hoc network. We have indicated how to map the parameter settings of both IEEE $802.11 \mathrm{~B}$ and IEEE $802.11 \mathrm{E}$ bottleneck nodes onto the fluid model, and the validation proves that the fluid modeling is accurate for both types of bottleneck nodes. It is also shown that the overall flow transfer time decreases by granting a larger share of the resource capacity to the bottleneck node, which can be obtained by an IEEE $802.11 \mathrm{E}$ bottleneck node.

Topics for further research include:

- Mathematical analysis of the fluid model with unequal resource sharing, i.e., $m_{n}>1$. This model is significantly harder to analyze than the model with $m_{n}=1$, where the resource sharing is entirely determined by the number of active sources; in case $m_{n}>1$ also the buffer content of the bottleneck node has to be taken into account.

- Alternative service disciplines at the bottleneck node. In the above analysis it is assumed that the packet scheduling at the bottleneck node is First Come First Serve. Alternative service disciplines, e.g. round robin, may yield considerably smaller mean overall flow transfer times.

- Investigation of the influence of higher-layer protocols, such as TCP, on the flow transfer time.

- A proper implementation of the unequal resource sharing policy $m_{n}=\infty$. Currently, obstacles for implementation are the lack of global knowledge of which nodes currently are bottlenecks and the absence of practical parameter settings to provide the desired resource sharing. A possible implementation is that each node is assigned an infinite $\mathrm{TXOP}_{\text {limit }}$ for all relay packets, i.e., a node sends all packets that it has to relay for other nodes in a single TxOP.

\section{Acknowledgements}

This work has been carried out partly in the SENTER-NOVEM funded project EASY WIRELESS and the Dutch BSIK/BRICKS project.

\section{References}

1. J.L. van den Berg, M.R.H. Mandjes and F. Roijers. Performance modeling of a Bottleneck Node in an IEEE 802.11 Ad-hoc Network. Proceedings of AdHoc-Now 2006, LNCS 4104, 321-336, 2006. Available as CWI-report at http://ftp.cwi.nl/ CWIreports/PNA/PNA-E0607.pdf. 
2. G. Bianchi. Performance analysis of the IEEE 802.11 Distributed Coordination Function. IEEE Journal on Selected Areas in Communications, 18: 535-547, 2000.

3. E.G. Coffman JR., R.R. Muntz, and H. Trotter. Waiting time distributions for Processor-Sharing systems. Journal of the ACM, 17: 123-130, 1970.

4. J.W. Cohen. The multiple phase service network with Generalized Processor sharing. Acta informatica, 12: 245-284, 1979.

5. C.H. Foh and M. Zukerman. Performance analysis of the IEeE 802.11 MAC protocol. Proceedings of European Wireless '02, Florence, Italy, 2002.

6. Z. Fu, P. Zerfos, H. Luo, S. Lu, L. Zhang, and M. Gerla. The impact of multihop wireless channel on TCP throughput and loss. Proceedings of INFOCOM '03, San Francisco, USA, 2003.

7. J. HE and H.K. Pung. Fairness of medium access control for multi-hop ad hoc networks. Computer Networks, 48: 867-890, 2005.

8. IEEE P802.11B/D7.0, Supplement: higher speed physical layer extension in the 2.4 GHz band, 1999.

9. IEEE P802.11E-2005, Amendment 8: Medium Access Control (MAC) Quality of Service Enhancements. November 2005.

10. R. Litjens, F. Roijers, J.L. van Den Berg, R.J. Boucherie, and M.J. Fleuren. Analysis of flow transfer times in IEEE 802.11 wireless LANs. Annals of Telecommunications, 59: 1407-1432, 2004.

11. F. Roijers, J.L. van Den Berg, X. FAn and M.J. Fleuren. A Performance Study on Service Integration in IEEE 802.11E wireless LANs. Computer Communications, 29: 2621-2633, 2006.

12. H.C. TiJms. Stochastic models: an algorithmic approach. Wiley \& Sons, 1994.

13. J. Zhao, Z. Guo, Q. Zhang, and W. Zhu. Performance Study of maC for Service Differentiation in IEEE 802.11. Proceeding of IEEE GLOBECOM: 33, 778 -782, 2002. 